\title{
Highly Water Stable Zirconium Metal-Organic Framework UiO-66 Membranes Supported on Alumina Hollow Fibers for Desalination
}

\author{
Xinlei Liu, Nilay Keser Demir, Zhentao Wu and Kang Li* \\ Department of Chemical Engineering, Imperial College London, London SW7 2AZ, United Kingdom
}

Supporting Information Placeholder

\begin{abstract}
In this study, continuous zirconium(IV) based metal-organic framework (Zr-MOF) membranes were prepared. The pure phase $\mathrm{Zr}-\mathrm{MOF}$ (i.e., UiO-66) polycrystalline membranes were fabricated on alumina hollow fibers using an in-situ solvothermal synthesis method. Single gas permeation and ion rejection tests were carried out to confirm the membrane integrity and functionality. The membrane exhibited excellent multivalent ion rejection (e.g., $86.3 \%$ for $\mathrm{Ca}^{2+}$, 98 . o\% for $\mathrm{Mg}^{2+}, 99.3 \%$ for $\mathrm{Al}^{+}$) based on size exclusion with moderate permeance (0.14 $\mathrm{L} \mathrm{m}^{-2} \mathrm{~h}^{-1}$ bar $\left.^{-1}\right)$ and good permeability $\left(0.28 \mathrm{~L} \mathrm{~m}^{-2} \mathrm{~h}^{-1} \mathrm{bar}^{-1} \mu \mathrm{m}\right)$. Benefiting from the exceptional chemical stability of the UiO-66 material, no degradation of the membrane performance was observed for various tests up to 170 hours towards a wide range of saline solutions. The high separation performance combined with its outstanding water stability suggests the developed UiO-66 membrane as a promising candidate for water desalination.
\end{abstract}

Metal-organic frameworks (MOFs), constructed by joining metal-containing units with organic linkers, ${ }^{1}$ have received tremendous attention due to their variety of potential applications including gas storage, ${ }^{1 c}, 1 \mathrm{~d}$ molecular separation, 1e heterogeneous catalysis if and smart sensor. 1g Given by their versatile architectures and customizable chemical functionalities, substantial advancements have been achieved in recent years, aiming at developing high performance polycrystalline MOF membranes. ${ }^{2}$ However, most of these membranes still suffer from the insufficient hydrothermal stability, 3 which precludes them to be applied in processes involving water.

Very recently, a series of zirconium(IV)-carboxylate MOFs (Zr-MOFs) with different topologies, a wide range of pore sizes and various functional groups have been emerged. 4 Benefiting from the strong coordination bonds between the hard acid-hard base interactions of the $\mathrm{Zr}(\mathrm{IV})$ atoms and carboxylate oxygens, this Zr-MOF family exhibits exceptional chemical and thermal stabilities. 4 To the best of our knowledge, so far, no continuous $\mathrm{Zr}-\mathrm{MOF}$ polycrystalline membranes have been reported. UiO-66 (UiO stands for University of Oslo) is a prototypical $\mathrm{Zr}-\mathrm{MOF}$, with the formula of $\mathrm{Zr}_{6} \mathrm{O}_{4}(\mathrm{OH})_{4}(\mathrm{BDC})_{6} \quad$ (BDC: 1,4-Benzenedicarboxylate), fcu topology and hydrophilic surface (Figure
S1). 4a, d The aperture size of the UiO-66 is $\sim 6.0 \AA$ as estimated from crystallographic data. 4a Thus, we can expect that membranes constructed by UiO-66 would achieve a high selectivity of $\mathrm{H}_{2} \mathrm{O}(\sim 2.8 \AA)$ over hydrated ions (6.6-9.5 $\AA$, 5 Table $\mathrm{S}_{1}$ ) based on size exclusion in water desalination. Membrane technology for water desalination is considered to be one of the most important solutions to the current worldwide water shortage. ${ }^{6}$

Herein, the development of continuous and high quality UiO-66 polycrystalline membranes supported on a predesigned porous alumina hollow fiber is presented. These membranes exhibited high multivalent ion rejection, moderate permeance and good permeability. Apart from providing a new candidate membrane for water desalination, successful development of the water stable $\mathrm{Zr}-\mathrm{MOF}$ membrane would also expect to speed up the paces towards practical applications of MOF membranes in near future.

The stability of UiO-66 was examined by suspending the as-synthesized samples in the water solutions. After test, the samples were thoroughly washed with water and dried

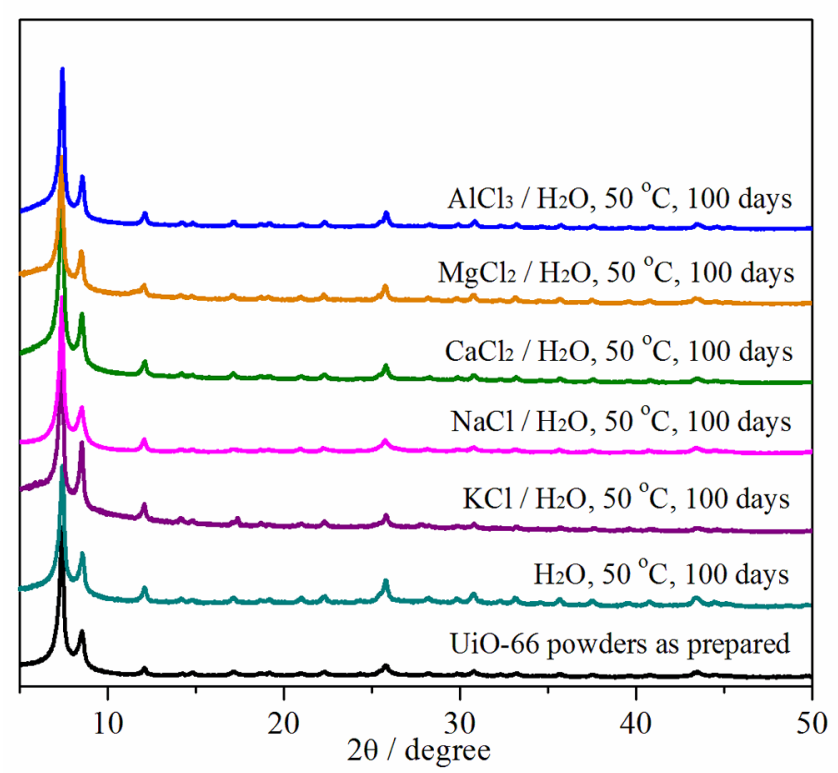

Figure 1. XRD patterns of UiO-66 powders as prepared and after stability test. The concentration of each saline solution is $0.20 w t$. \%. 

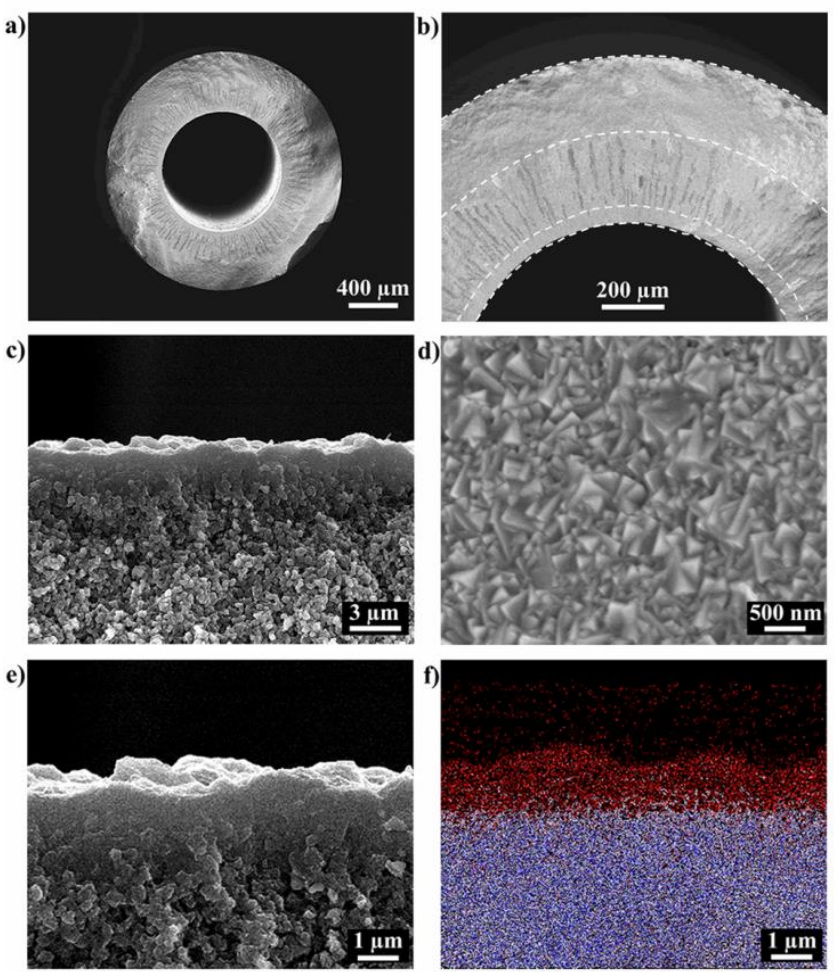

Figure 2. SEM images (a, b, c, e: cross section; d: top view) and EDXS mapping (Figure $2 \mathrm{f}$, corresponding to the Figure 2e. $\mathrm{Zr}$ signal: red; $\mathrm{Al}$ signal: light blue) of the alumina hollow fiber (HF) supported UiO-66 membranes. The membranes were fabricated on the outer surface of the HF.

before characterization. As shown in Figure 1, the UiO-66 exhibits excellent stabilities in both DI water and various saline water solutions. After 100 days of continuous stability test under the hydrothermal conditions, the good crystallinity of UiO-66 samples was retained, although slight differences of the peak strength ratio can be observed in the XRD patterns. Due to the 2 Theta errors in the XRD instrumentation, the diffraction peaks of UiO-66 shifted slightly after these treatments. To further demonstrate the stability of UiO-66, $\mathrm{N}_{2}$ adsorption experiments were conducted. The adsorption isotherms (Figure S2) indicate that the porosity of UiO-66 is perfectly maintained in saline water solutions. Moreover, during the test, no morphology changes and ion exchange reactions occurred on UiO-66 as evidenced by the SEM images (Figure $\mathrm{S}_{3}$ ) and EDXS analysis (Figure $\mathrm{S}_{4}, \mathrm{~S}_{5}$ ), respectively.

In light of the above properties, we used the UiO-66 as a material to fabricate membranes for water desalination. Porous ceramic hollow fibers (HF) employed as substrates were preferred due to their low transport resistance, high packing density, easy scaling up, and good chemical and thermal stability. ${ }^{7}$ As shown in Figures 2a, 2b, and Figure S6, the alumina HF used in this study contains two sponge-like layers sandwiching a layer of finger-like voids. The UiO-66 membrane was synthesized on the outer surface of such HF substrate by an in-situ solvothermal synthesis method (Figure $\mathrm{S}_{7}$ ). The successful deposition of a well intergrown UiO-66 polycrystalline layer on the HF was accomplished after a sufficient optimization of the preparation parameters and conditions, including the concentrations of metal and ligand, the amount of water and modulators in the mother solution, the synthesis duration and temperature as well as the types of solvents and microstructures of hollow fibers. The optimized recipe is given here: $\mathrm{ZrCl}_{4}, 1,4$-benzenedicarboxylic acid and DI water were dissolved in $60 \mathrm{~mL}$ DMF under stirring to give a molar composition: $\mathrm{Zr}^{4+} / \mathrm{BDC} / \mathrm{H}_{2} \mathrm{O} / \mathrm{DMF}=1: 1: 1: 500$. This clear solution was transferred into a Teflon-lined stainless steel autoclave in which an alumina hollow fiber (O.D.: $2.1 \mathrm{~mm}$, length: 6o $\mathrm{mm}$ ) was placed vertically with both ends sealed (Figure $\mathrm{S}_{7}$ ). Afterwards the autoclave was placed in a convective oven and heated at $120^{\circ} \mathrm{C}$ for 3 days. After cooling, the membrane was washed with ethanol and dried at $25^{\circ} \mathrm{C}$ overnight under vacuum. To guarantee a good reproducibility, it should be noted that anhydrous chemicals and solvent should be kept fresh and handled with care to avoid deliquescence or moisture sorption. This is because the amount of water in the mother solution for membrane synthesis is critical to the nucleation and intergrowth of UiO-66 crystals.

After solvothermal synthesis, a continuous polycrystalline UiO-66 membrane was formed on the HF without any visible cracks or pinholes as shown in Figure $2 \mathrm{c}$ and $2 \mathrm{~d}$. The UiO-66 grains are approximately $0.2-0.6 \mu \mathrm{m}$ in size and well intergrown, forming the membrane of around $2.0 \mu \mathrm{m}$ in thickness, substantially thinner than most of the MOF membranes reported thus far. 2,3 A sharp transition between the UiO-66 membrane layer ( $\mathrm{Zr}$ signal) and alumina substrate (Al signal) can be observed from the EDXS mapping image (Figure $2 f$, corresponding to the Figure 2e), revealing that no detectable $\mathrm{UiO}-66$ crystals nucleated into the bulk HF. The XRD pattern (Figure S8) indicates that this UiO-66 membrane consists of grains with no perfect preferred orientations and is free of impurity phases. Generally, to prepare satisfying MOF membranes, seedingsecondary growth method and substrate surface modification method are used. 3a In this study, high quality UiO-66 membrane was prepared by in-situ synthesis method. This could be because the BDC ligand acted as a surface modifier of the substrate during the synthesis via constructing coordination bonds between the carboxylate oxgens and aluminum atoms from substrate. ${ }^{2 g}$ So, the nucleation and growth of UiO-66 on the alumina substrate were promoted to a large extent.

For separation applications, a defect-free polycrystalline membrane layer is required to achieve high separation performance. Single gas permeation test is one of the best ways to assess the integrity of polycrystalline membranes. ${ }^{2 h}$, $2 \mathrm{i}, 2 \mathrm{k}, 2 \mathrm{~m}, 2 \mathrm{r}, 2 \mathrm{~s}$ The gas permeation through the bare HF and the as-synthesized UiO-66 membrane was measured by a soapfilm flowmeter at $20 \pm 2{ }^{\circ} \mathrm{C}$ under the pressure difference across the membrane of 1.0 bar. The bare HF substrate showed an $\mathrm{H}_{2}$ permeance of $1.5 \times 10^{-4} \mathrm{~mol} \mathrm{~m}^{-2} \mathrm{~s}^{-1} \mathrm{~Pa}^{-1}$ with a low $\mathrm{H}_{2} / \mathrm{N}_{2}$ ideal selectivity ( 2.5). Ideal selectivity is defined as the permeance ratio of gases $\mathrm{A}$ and $\mathrm{B}$. For the UiO-66 membrane (Figure S9), it can be seen that the permeances did not depend on the kinetic diameters of the gases since the aperture size of $\mathrm{UiO}-66(\sim 6.0 \AA){ }^{4 a}$ is much bigger $(2.9 \AA$, $3.3 \AA$, $3.6 \AA$, $3.8 \AA$ for $\mathrm{H}_{2}, \mathrm{CO}_{2}, \mathrm{~N}_{2}$, and $\mathrm{CH}_{4}$, respectively ${ }^{8}$ ). The $\mathrm{H}_{2}$ permeance was approximately $7.2 \times 10^{-7} \mathrm{~mol} \mathrm{~m}^{-2} \mathrm{~s}^{-1}$ $\mathrm{Pa}^{-1}$ and the ideal selectivity of $\mathrm{H}_{2} / \mathrm{N}_{2}$ and $\mathrm{H}_{2} / \mathrm{CH}_{4}$ was 22.4 and 6.4, respectively, which was much higher than the values of Knudsen diffusion ratio ( 3.7 and 2.8 , respectively). This indicates the membrane itself shows good quality and thus 


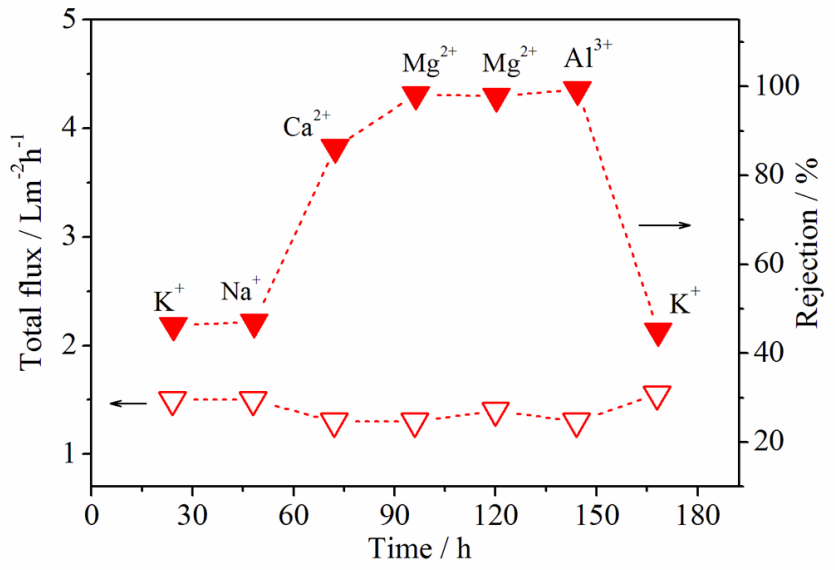

Figure 3. Desalination performance of the UiO-66 membrane. Five different saline water solutions (containing $\mathrm{KCl}, \mathrm{NaCl}, \mathrm{CaCl}_{2}, \mathrm{MgCl}_{2}$ or $\mathrm{AlCl}_{3}$ ) with the same concentration (o.20wt. \%) were applied as feeds at $20 \pm 2{ }^{\circ} \mathrm{C}$ under a pressure difference of 10.0 bar. The order of water and hydrated ion diameters is: $\mathrm{H}_{2} \mathrm{O}(2.8 \AA)<\mathrm{Cl}^{-}(6.6 \AA) \sim \mathrm{K}^{+}$ $(6.6 \AA)<\mathrm{Na}^{+}(7.2 \AA)<\mathrm{Ca}^{2+}(8.2 \AA)<\mathrm{Mg}^{2+}(8.6 \AA)<\mathrm{Al}^{++}(9.5$ A). 5

its integrity was confirmed. The permeance of $\mathrm{CO}_{2}\left(9.5 \times 10^{-7}\right.$ mol m$\left.~_{-2} \mathrm{~s}^{-1} \mathrm{~Pa}^{-1}\right)$ is very high, leading to a good separating selectivity (e.g., 29.7 for $\mathrm{CO}_{2} / \mathrm{N}_{2}$, Figure $\mathrm{S} 9$, S1o). This finding is easily understood because $\mathrm{UiO}-66$ can preferentially adsorb $\mathrm{CO}_{2}$ over other gases due to the specific interaction of $\mathrm{CO}_{2}$ with the hydroxylated $\mathrm{Zr}_{6}$ cluster in the framework. 9

The desalination performance of the bare $\mathrm{HF}$ and $\mathrm{UiO}-66$ membrane was carried out in a dead-end system (Figure S11) at $20 \pm 2{ }^{\circ} \mathrm{C}$ under a transmembrane pressure of 10.0 bar. Five different saline water solutions (containing $\mathrm{KCl}, \mathrm{NaCl}, \mathrm{CaCl}_{2}$, $\mathrm{MgCl}_{2}$ or $\mathrm{AlCl}_{3}$ ) with the same concentration (o.2owt. \%) were utilized as feeds. The rejections were determined by analyzing the concentrations of the ions in the retentate and permeate using ionic conductivity. The total flux of bare HF was $1.3 \times 10^{4} \mathrm{~L} \mathrm{~m}^{-2} \mathrm{~h}^{-1}$ with no ion rejection. As shown in Figure 3, for the UiO-66 membrane, the ion rejection increased with increasing hydrated diameter of ions demonstrating a size selective diffusion in the desalination process. Although the aperture size of UiO-66 was estimated from crystallographic data to be $\sim 6.0 \AA$, ${ }^{\text {a }}$ the rejections for monovalent ions $\left(\mathrm{Cl}^{-}\right.$: $6.6 \AA, \mathrm{K}^{+}: 6.6 \AA \mathrm{Na}^{+}: 7.2 \AA$ ) 5 were moderate, i.e., $45.7 \%$ and $47.0 \%$ for $\mathrm{K}^{+}$and $\mathrm{Na}^{+}$, respectively. One possible reason for this phenomenon could be in virtue of the ligand dynamics of UiO-66 ${ }^{\text {10a }}$ since its carboxylate groups can change their coordination mode from edge-bridging to monodentate. 4a While a second reason could be due to the missing-ligand defects ${ }^{10 b}, 10 \mathrm{c}$ in the UiO-66 crystals. That is why o-xylene molecules with kinetic diameter around $7.4 \AA$ can pass through the apertures of $\mathrm{UiO}-66$, which was reported elsewhere. ${ }^{\text {od }}$ The membrane exhibited very high rejections for multivalent and trivalent cations $(86.3 \%, 98.0 \%, 99.3 \%$ for $\mathrm{Ca}^{2+}(8.2 \AA), \mathrm{Mg}^{2+}(8.6 \AA)$ and $\mathrm{Al}^{3+}(9.5 \AA)$, respectively) because these hydrated ion sizes might have reached or exceeded the effective aperture size of UiO-66. These results further prove that the as-synthesized polycrystalline membrane is a continuous membrane with high quality. The membrane flux and pressure normalized flux (i.e., permeance) are moderate, around $1.4 \mathrm{~L} \mathrm{~m}^{-2} \mathrm{~h}^{-1}$ and $0.14 \mathrm{~L} \mathrm{~m}^{-2}$ $\mathrm{h}^{-1}$ bar $^{-1}$, respectively. The thickness-normalized water permeance (i.e., permeability) of the UiO-66 membrane was determined to be $0.28 \mathrm{~L} \mathrm{~m}^{-2} \mathrm{~h}^{-1} \mathrm{bar}^{-1} \mu \mathrm{m}$ based on a measured membrane thickness of $2.0 \mu \mathrm{m}$. This value is the same order of magnitude as the reported water permeabilities of commercial polymeric reverse osmosis (RO) and nanofiltration (NF) membranes (0.047-0.72 $\mathrm{L} \mathrm{m}^{-2} \mathrm{~h}^{-1} \mathrm{bar}^{-1} \mu \mathrm{m}$, Table S2), 6 demonstrating the MOF UiO-66 is a good membrane material for water treatment. If polycrystalline UiO-66 membrane with much thinner thickness can be prepared via developing advanced membrane fabrication technologies, a higher membrane flux will be achieved.

As desired, this membrane shows very stable water filtration performance (Figure 3) benefiting from the exceptional stability of UiO-66 material. No discernible degradation of the membrane performance was observed during the tests around 170 hours with different saline solutions at the transmembrane pressure of 10.0 bar. This indicates that pore blockage of UiO-66 and damage of the crystal structure and grain boundaries did not occur during the test. The structure and morphology of the UiO-66 membrane remained unchanged after the separation test as evidenced by the XRD (Figure S12) and SEM (Figure S13) characterizations, respectively. After flushed with DI water, no salt residues on the membrane can be observed according to the element analysis data (Figure S13). The good stability together with outstanding membrane performance strongly encourages us to use $\mathrm{UiO}-66$ as a next generation membrane for water softening in seawater desalination.

In summary, a continuous polycrystalline Zr-MOF UiO-66 membrane was successfully fabricated on porous ceramic hollow fibers by employing an in-situ solvothermal synthesis method. These membranes exhibited high multivalent ion rejection (e.g., 86.3\% for $\mathrm{Ca}^{2+}$, 98. o\% for $\mathrm{Mg}^{2+}$, 99.3\% for $\left.\mathrm{Al}^{+}\right)$based on size exclusion mechanism with moderate permeance ( $0.14 \mathrm{~L} \mathrm{~m}^{-2} \mathrm{~h}^{-1}$ bar $^{-1}$ ) and good permeability $(0.28 \mathrm{~L}$ $\mathrm{m}^{-2} \mathrm{~h}^{-1}$ bar $\left.^{-1} \mu \mathrm{m}\right)$ for water desalination. Furthermore, the membranes have very stable water filtration performance because of the exceptional chemical stability of UiO-66 material. This high separation performance combined with its outstanding stability suggests the developed UiO-66 membrane as a promising candidate for water desalination. Our studies are in progress of exploring new substrates and fabrication methods for further developing ultrathin UiO-66 membranes aiming for cross-flow conditions, normally used in practical applications.

Since a series of exceptional chemical and thermal stable Zr-MOF materials with a wide range of pore sizes and various functional groups are already available, 4 there would be some optimal preparation conditions where the membranes based on this $\mathrm{Zr}-\mathrm{MOF}$ analogue can be prepared for use in real industrial separation processes such as gas separation, pervaporation and water treatment (e.g., pressure driven desalination) in the future.

\section{ASSOCIATED CONTENT}

\section{Supporting Information}

Experimental and characterization details; structure of UiO66; $\mathrm{N}_{2}$ adsorption isotherms, SEM images, EDXS data and XRD patterns of UiO-66 powders and membranes; SEM image and pore size distribution of alumina hollow fibers; schematic and flow diagrams; comparison of membrane 
performances. This material is available free of charge via the Internet at http://pubs.acs.org.

\section{AUTHOR INFORMATION}

\section{Corresponding Author}

kang.li@imperial.ac.uk

Notes

The authors declare no competing financial interests.

\section{ACKNOWLEDGMENT}

We acknowledge the grant from Engineering and Physical Sciences Research Council (EPSRC, EP/Jo14974/1).

\section{REFERENCES}

(1) (a) Zhou, H. C.; Long, J. R.; Yaghi, O. M. Chem. Rev. 2012, 112, 673. (b) Furukawa, H.; Cordova, K. E.; O’Keeffe, M.; Yaghi, O. M. Science 2013, 341, 1230444. (c) Férey, G. Chem. Soc. Rev. 2008, 37, 191. (d) Kitagawa, S.; Kitaura, R.; Noro, S. Angew. Chem. Int. Ed. 2oo4, 43, 2334. (e) Li, J. R.; Sculley, J.; Zhou, H. C. Chem. Rev. 2012, 112, 869. (f) Liu, J.; Chen, L.; Cui, H.; Zhang, J.; Zhang, L.; Su, C. Y. Chem. Soc. Rev. 2014, 43, 6o11. (g) Kreno, L. E.; Leong, K.; Farha, O. K.; Allendorf, M.; Van Duyne, R. P.; Hupp, J. T. Chem. Rev. 2012, 112, 1105.

(2) (a) Guo, H.; Zhu, G.; Hewitt, I. J.; Qiu, S. J. Am. Chem. Soc. 2009, 131, 1646. (b) Bux, H.; Liang, F.; Li, Y.; Cravillon, J.; Wiebcke, M.; Caro, J. J. Am. Chem. Soc. 2009, 131, 160oo. (c) Ranjan, R.; Tsapatsis, M. Chem. Mater. 2009, 21, 4920. (d) Li, Y. S.; Liang, F. Y.; Bux, H.; Feldhoff, A.; Yang, W. S.; Caro, J. Angew. Chem. Int. Ed. 2010, 49, 548. (e) Gascon, J.; Kapteijn, F. Angew. Chem. Int. Ed. 2010 49, 1530. (f) Venna, S. R.; Carreon, M. A. J. Am. Chem. Soc. 2010, 132, 76. (g) Hu, Y.; Dong, X.; Nan, J.; Jin, W.; Ren, X.; Xu, N.; Lee, Y. M. Chem. Commun. 2011, 47, 737. (h) Yao, J.; Dong, D.; Li, D.; He, L.; Xu, G.; Wang, H. Chem. Commun. 2011, 47, 2559. (i) Pan, Y.; Lai, Z. Chem. Commun. 2011, 47, 10275. (j) Shekhah, O.; Liu, J.; Fischer, R. A.; Wöll, C. Chem. Soc. Rev. 2011, 40, 1081. (k) Brown, A. J.; Johnson, J. R.; Lydon, M. E.; Koros, W. J.; Jones, C. W.; Nair, S. Angew. Chem. Int. Ed. 2012, 51, 10615. (l) Gascon, J.; Kapteijn, F.; Zornoza, B.; Sebastián, V.; Casado, C.; Coronas, J. Chem. Mater. 2012, 24, 2829. (m) Liu, Q.; Wang, N.; Caro, J.; Huang, A. J. Am. Chem. Soc. 2013, 135, 17679. (n) Dong, X.; Lin, Y. Chem. Commun. 2013, 49, 1196. (o) Kwon, H. T.; Jeong, H. K. J. Am. Chem. Soc. 2013, 135, 10763. (p) Brown, A. J.; Brunelli, N. A.; Eum, K.; Rashidi, F.; Johnson, J. R.; Koros, W. J.; Jones, C. W.; Nair, S. Science 2014, 345, 72. (q) Peng, Y.; Li, Y.; Ban, Y.; Jin, H.; Jiao, W.; Liu, X.; Yang, W. Science 2014, 346, 1356. (r) AlMaythalony, B. A.; Shekhah, O.; Swaidan, R.; Belmabkhout, Y.;
Pinnau, I.; Eddaoudi, M. J. Am. Chem. Soc. 2015, 137, 1754. (s) Liu, Y.; Pan, J. H.; Wang, N.; Steinbach, F.; Liu, X.; Caro, J. Angew. Chem. Int. Ed. 2015, 54, 3028.

(3) (a) Qiu, S.; Xue, M.; Zhu, G. Chem. Soc. Rev. 2014, 43, 6116. (b) Lin, Y. Curr. Opin. Chem. Eng. 2015, 8, 21.

(4) (a) Cavka, J. H.; Jakobsen, S.; Olsbye, U.; Guillou, N.; Lamberti, C.; Bordiga, S.; Lillerud, K. P. J. Am. Chem. Soc. 20o8, 130, 1385o. (b) Guillerm, V.; Ragon, F.; Dan-Hardi, M.; Devic, T.; Vishnuvarthan, M.; Campo, B.; Vimont, A.; Clet, G.; Yang, Q.; Maurin, G.; Férey, G.; Vittadini, A.; Gross, S.; Serre, C. Angew. Chem. Int. Ed. 2012, 51, 9267. (c) Mondloch, J. E.; Bury, W.; Fairen-Jimenez, D.; Kwon, S.; DeMarco, E. J.; Weston, M. H.; Sarjeant, A. A.; Nguyen, S. T.; Stair, P. C.; Snurr, R. Q.; Farha, O. K.; Hupp, J. T. J. Am. Chem. Soc. 2013, 135, 10294. (d) Furukawa, H.; Gándara, F.; Zhang, Y. B.; Jiang, J.; Queen, W. L.; Hudson, M. R.; Yaghi, O. M. J. Am. Chem. Soc. 2014, 136, 4369. (e) Kalidindi, S. B.; Nayak, S.; Briggs, M. E.; Jansat, S.; Katsoulidis, A. P.; Miller, G. J.; Warren, J. E.; Antypov, D.; Corà, F.; Slater, B.; Prestly, M. R.; Martí-Gastaldo, C.; Rosseinsky, M. J. Angew. Chem. Int. Ed. 2015, 54, 221. (f) Liu, T. F.; Feng, D.; Chen, Y. P.; Zou, L.; Bosch, M.; Yuan, S.; Wei, Z.; Fordham, S.; Wang, K.; Zhou, H. C. J. Am. Chem. Soc. 2015, 137, 413.

(5) Nightingale Jr., E. R. J. Phys. Chem. 1959, 63, 1381.

(6) (a) Elimelech, M.; Phillip, W. A. Science 2011, 333, 712. (b) Greenlee, L. F.; Lawler, D. F.; Freeman, B. D.; Marrot, B.; Moulin, P. Water Res. 2009, 43, 2317. (c) Pendergast, M. M.; Hoek, E. M. V. Energy Environ. Sci. 2011, 4, 1946. (d) Zhou, M.; Nemade, P. R.; Lu, X.; Zeng, X.; Hatakeyama, E. S.; Noble, R. D.; Gin, D. L. J. Am. Chem. Soc. 2007, 129, 9574. (e) Lee, K. P.; Aront, T. C.; Mattia, D. J. Membr. Sci. 2011, 370, 1 .

(7) (a) Othman, M. H. D.; Droushiotis, N.; Wu, Z.; Kelsall, G.; Li, K. Adv. Mater. 2011, 23, 2480. (b) Wu, Z.; Faiz, R.; Li, T.; Kingsbury, B. F. K.; Li, K. J. Membr. Sci. 2013, 446, 286. (c) Lee, M.; Wu, Z.; Wang, R.; Li, K. J. Membr. Sci. 2014, 461, 39. (d) Wang, Z.; Ge, Q.; Shao, J.; Yan, Y. J. Am. Chem. Soc. 20o9, 131, 6910. (e) Shao, J.; Zhan, Z.; Li, J.; Wang, Z.; Li, K.; Yan, Y. J. Membr. Sci. 2014, 451, 10.

(8) Breck, D. W. Zeolite Molecular Sieves: Structure, Chemistry and Use. John Wiley \& Sons, Inc.: New York, 1974.

(9) Cmarik, G. E.; Kim, M.; Cohen, S. M.; Walton, K. S. Langmuir 2012, 28, 15606 .

(10) (a) Devautour-Vinot, S.; Martineau, C.; Diaby, S.; Ben-Yahia, M.; Miller, S.; Serre, C.; Horcajada, P.; Cunha, D.; Taulelle, F.; Maurin, G. J. Phys. Chem. C 2013, 117, 11694. (b) Valenzano, L.; Civalleri, B.; Chavan, S.; Bordiga, S.; Nilsen, M. H.; Jakobsen, S.; Lillerud, K. P.; Lamberti, C. Chem. Mater. 2011, 23, 1700. (c) Wu, H.; Chua, Y. S.; Krungleviciute, V.; Tyagi, M.; Chen, P.; Yildirim, T.; Zhou, W. J. Am. Chem. Soc. 2013, 135, 10525. (d) Chang, N.; Yan, X. P. J. Chromatogr. A 2012, 1257, 116. 


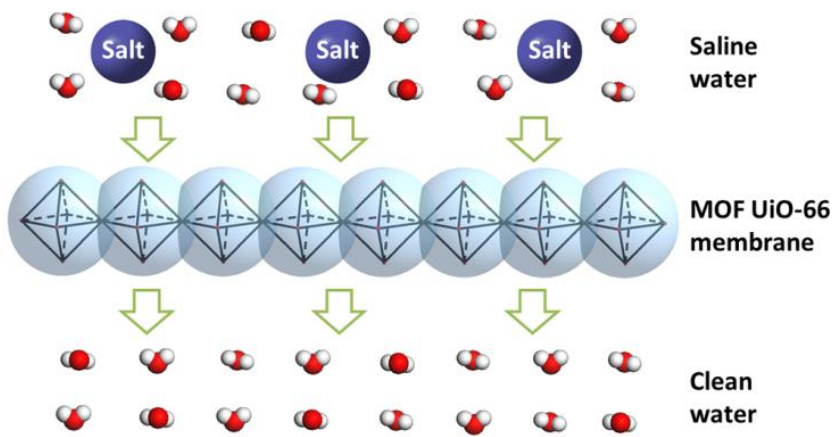

